\title{
The ARGOS vibration compensation system
}

\author{
Matthias Rosensteiner ${ }^{\mathrm{a} *}$ Diethard Peter ${ }^{\mathrm{b}}$, Wolfgang Gässler ${ }^{\mathrm{b}}$ and Julian Ziegleder ${ }^{\mathrm{a}}$ \\ ${ }^{a}$ Max Planck Institute for Extraterrestrial Physics \\ b Max Planck Institute for Astronomy
}

\begin{abstract}
Vibrations are one of the biggest problems in astronomical instrumentation. Even after carefully designing an adaptive optics system, it remains a major contribution to the residual error. ARGOS is the facility GLAO system for the LBT. It has a dedicated vibration compensation system to stabilize the laser guide stars, which allows operation in windy conditions. We give an overview of the setup and discuss its performance and impact on the reliability of ARGOS.
\end{abstract}

\section{Introduction}

In the last years the ARGOS[2] ground layer adaptive optics instrument has been installed at the Large Binocular Telescope (LBT). The goal of the system is to reduce the seeing by a factor of two or better for the imager and spectrograph LUCI $[5,1]$. The system consists for each side of the LBT of three Rayleigh laser guide stars that are produced by gating a pulsed laser with Pockels cells. The laser light is fed into high order wavefront sensors.

The system is required to work under all reasonable seeing and wind conditions. Therefore, a good stability of the laser beacon on sky, even under the influence of wind shake is necessary. The vibration compensation system $[3,4]$ is designed to detect vibrations in the laser uplink path and mitigate the jitter of the beacon. This paper gives an overview of the system and vibration features we found. We show its performance with respect to the improvement of the stability of the laser beacon on the wavefront sensors. This allows for observations under stronger wind and helps the robustness of ARGOS.

\section{The vibration compensation system}

The lasers of the ARGOS system are launched from the central platform of the telescope, between the two primary mirrors. At the top of the wind brace the light is deflected with two flat mirrors (LM1 and LM2) behind the adaptive secondary mirrors and then propagated to the sky.

On the backside of each of the LM mirrors four accelerometers are installed to measure the vibrations in the system. The signals are used to control a piezo mirror in open loop to counter the vibrations on the LMs. The Kalman filter based control is computed in a NI real-time system. Additionally, a link from the wavefront sensor provides jitter information for fast guiding.

\footnotetext{
${ }^{*}$ Corresponding author: rosensteiner@mpe.mpg.de
} 
For our test we took vibration data on two different ARGOS runs in March and May 2017. During the nights we recorded snapshots of vibration data with a length of one minute each. This includes the measurements of the accelerometers, the computed feed forward control, the piezo mirror position and the jitter information from the wavefront sensors. This way we obtained data for various observing conditions: different wind speeds, telescope elevations and seeing.

\section{Analysis of vibration lines}



Figure 1: Vibration spectra of accelerometer on LM1 (SX side); averaged measurements, March 2017; x: frequency $(\mathrm{Hz}), \mathrm{y}$ : accelerometer output

In our analysis we first want to have a look at the signal we get from the accelerometers. In Figure 1 we can see the spectra of the four accelerometers on LM1 (SX side), averaged over all our measurements from March. In the spectra of the vibration signal several features can be found: for some of the lines, we can identify interesting correlations. The figures of specific lines can be read as follows: the $\mathrm{y}$ axis are the different data sets, the $\mathrm{x}$ axis gives the frequency, cut around the vibration line, for each of the four accelerometers next to each other.

Some lines (e.g. $18 \mathrm{~Hz}$, see Fig. 2) are strongly wind dependent, which might correspond to wind pressure excited resonances in the mirrors or the telescope structure itself. It can be seen that the vibration has a comparable strength on all accelerometers. Other lines are dependent on the elevation of the telescope, either getting stronger with lower $(28.5 \mathrm{~Hz}$, see Fig. 3) or with higher (97.5 Hz, see Fig. 4) elevation. This indicates that the vibrations are self-made in the telescope structure, for example origins in some pump, fans, etc. and could, if the source can be identified, be eliminated. 


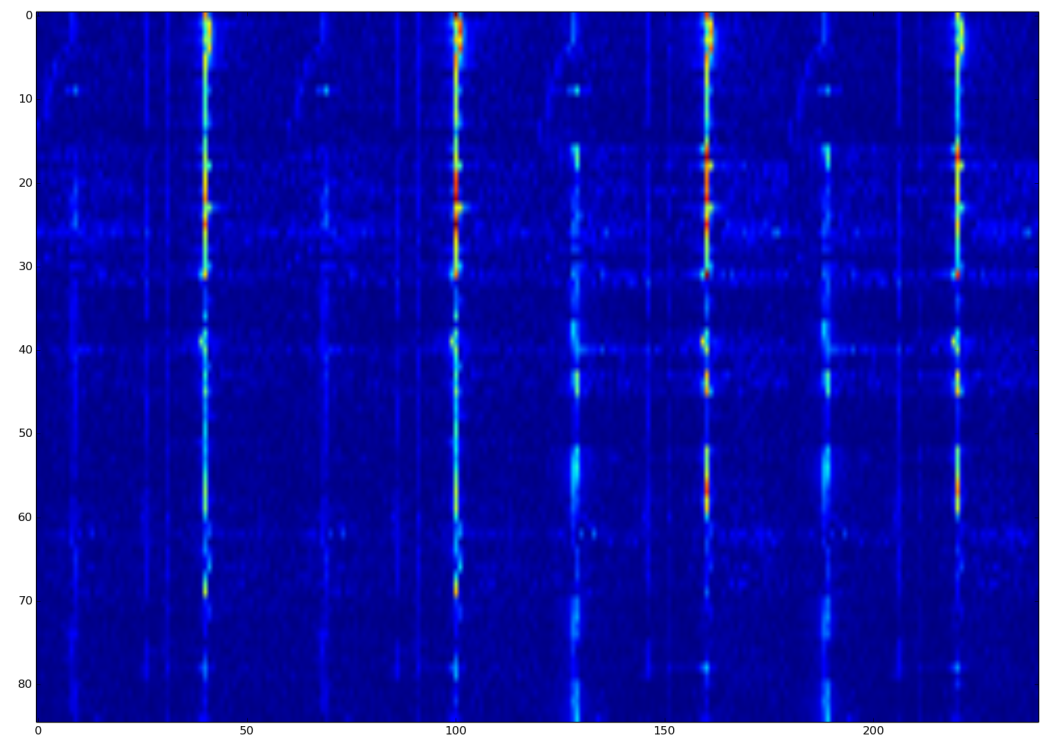

Figure 2: Frequency $18 \mathrm{~Hz}$ line for all 4 accelerometers on LM1 (SX); x: frequency cuts around the line for all four accelerometers; y: different data sets

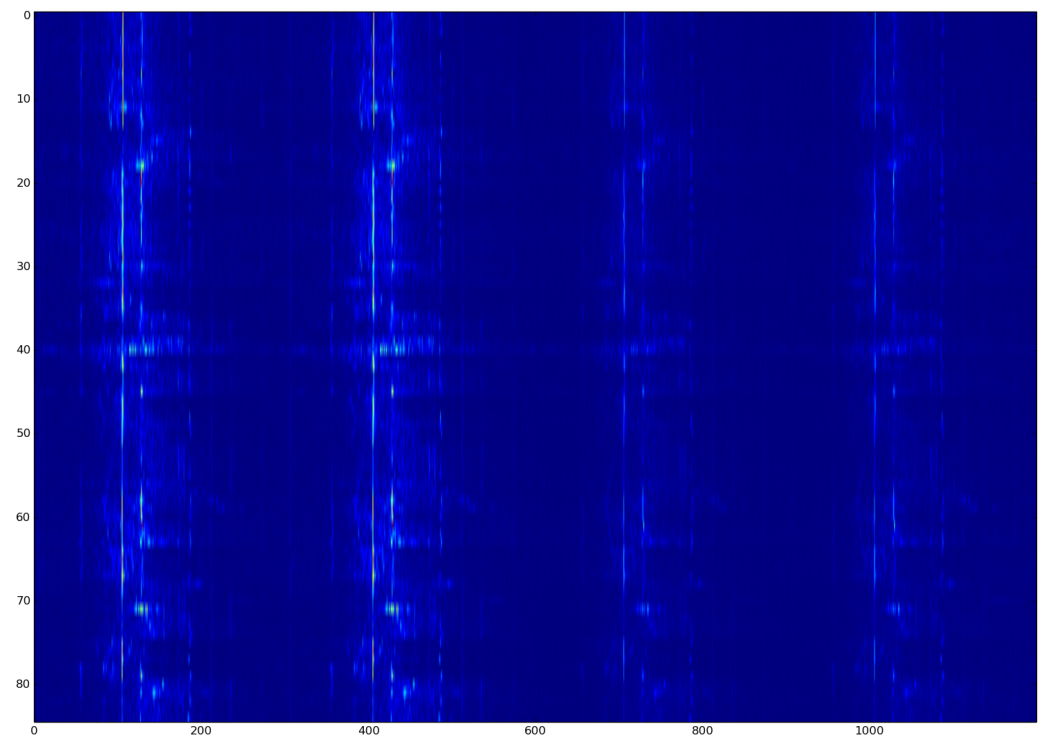

Figure 3: Frequency $28.5 \mathrm{~Hz}$ line for all 4 accelerometers on LM1 (SX); x: frequency cuts around the line for all four accelerometers; y: different data sets 


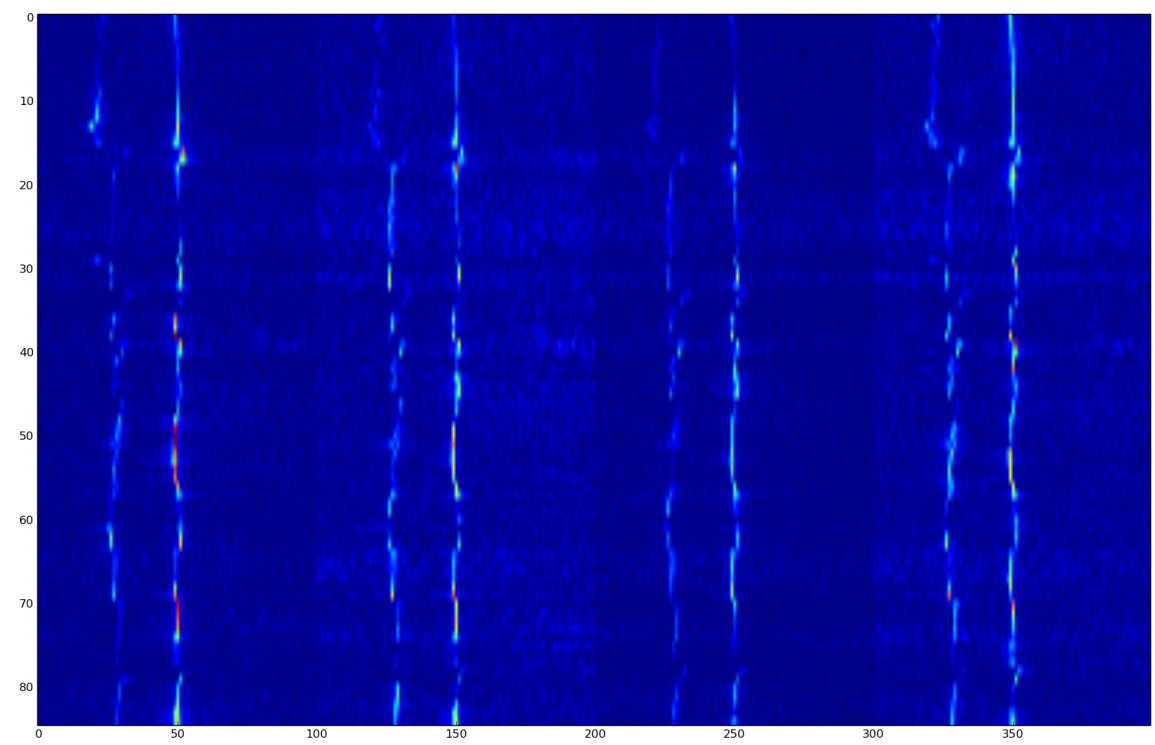

Figure 4: Frequency $97.5 \mathrm{~Hz}$ line for all 4 accelerometers on LM1 (SX); x: frequency cuts around the line for all four accelerometers; y: different data sets

\section{Performance of the vibration compensation}

For the evaluation of the performance of the vibration compensation we used the vibration data from the wavefront sensors. With this measurements we analyze the stability of the laser beacon on the detector. We used night time with medium weather conditions $(7 \mathrm{~m} / \mathrm{s}$ wind, 1.3 arcsec seeing) to test the impact of the compensation system. For several minutes the compensation was turned on or off every 30 seconds. For the recorded data we computed the rms jitter error in the wavefront sensor.

In Figure 5 we show the strength of the jitter for each second of data. The impact of the vibration compensation is clearly visible in the plot, the 30 seconds periods, when the control is turned on or off, easily distinguishable. This is a good indication that the system compensates for the vibrations in the laser uplink path correctly. Note, that the data includes the uncorrected vibration of the downlink path as well. Table 1 shows the average rms error for both states for each side and axis and additionally gives the relative improvement of the jitter stability on the wavefront sensors.

The vibration on one axis (SX, $\mathrm{x}$ ) is clearly worse than on the other ones. This can also be seen in independent measurements of other instruments on the LBT and needs further investigations.

\begin{tabular}{lrrrr} 
& SX, x & SX, y & DX, x & DX, y \\
\hline vibration compensation off (mas) & 370 & 200 & 180 & 145 \\
vibration compensation on (mas) & 230 & 120 & 120 & 110 \\
relative residual & $63 \%$ & $60 \%$ & $67 \%$ & $78 \%$
\end{tabular}

Table 1: RMS jitter error with and without vibration compensation 


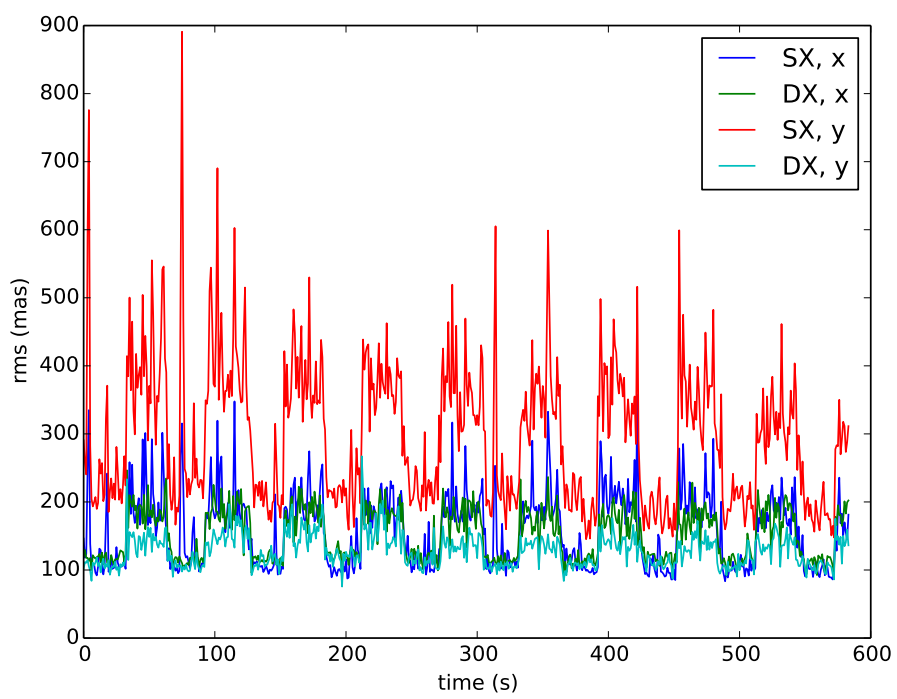

Figure 5: Jitter error with the vibration compensation turned on and off every 30 seconds

\section{Improvement of the fast guiding link}

The accelerometers are most sensitive at high frequencies. In order to get a good compensation for the lower frequencies, the jitter information is used from the wavefront sensors. The jitter information is added in the realtime control computer of the vibration compensation system to the control signal computed from the accelerometer data. But especially for frequencies in the middle range $(\tilde{6} 0 \mathrm{~Hz})$ the delay of this sequence is too high for a good correction. To improve the delay of this closed loop control, a direct summation device is added to the system to bypass the realtime control computer. As a result the delay is improved by $1 \mathrm{~ms}$.

In Figure 6 the vibration spectrum of the old configuration is compared to the new setup. It can be observed that the vibrations in the medium frequency range of about $50-70 \mathrm{~Hz}$ are improved with the help of the direct summation. As a comparison with the changing conditions during the different runs is difficult, we plotted the performance of the two configurations with respect to the wind speed in Figure 7. The improvement of the jitter can be clearly seen, so the direct summation further helps the stability of the system.

\section{Conclusion}

The ARGOS vibration compensation system stabilizes the laser uplink path with the help of accelerometers. In this paper we have shown that there are interesting features in the vibration spectra on the laser path fold mirrors. These could be used to increase the stability of the LBT as a whole. The improvement of the jitter on the wavefront sensors with the open loop vibration compensation is clearly visible. Together with an upgrade in the fast guiding loop this helps to run the ARGOS GLAO loop reliably in windy conditions 

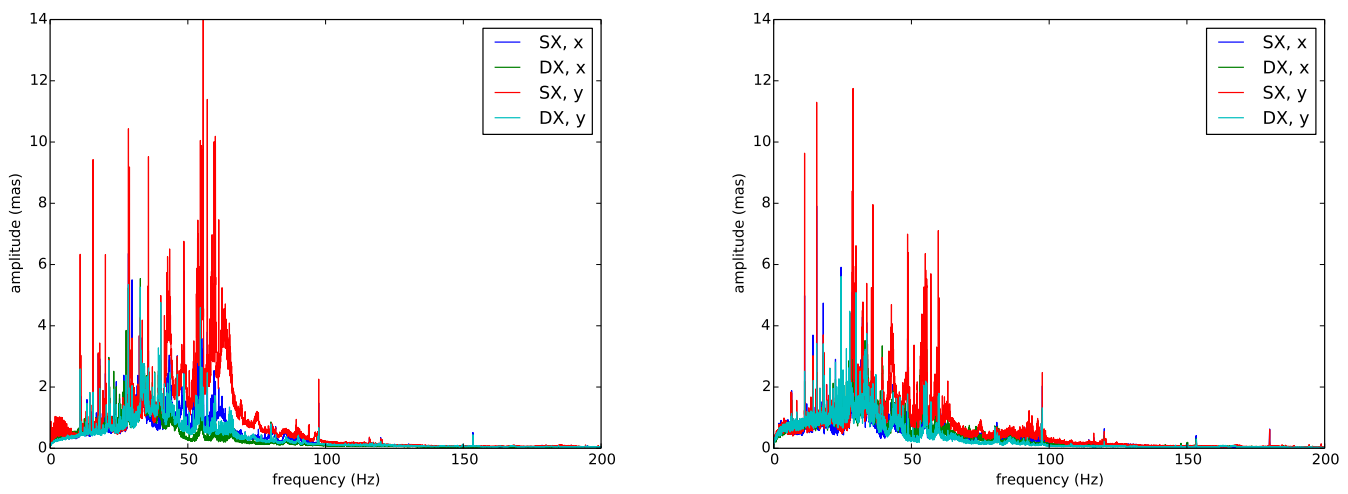

Figure 6: Vibration spectrum before (left) and after (right) the fast link improvement

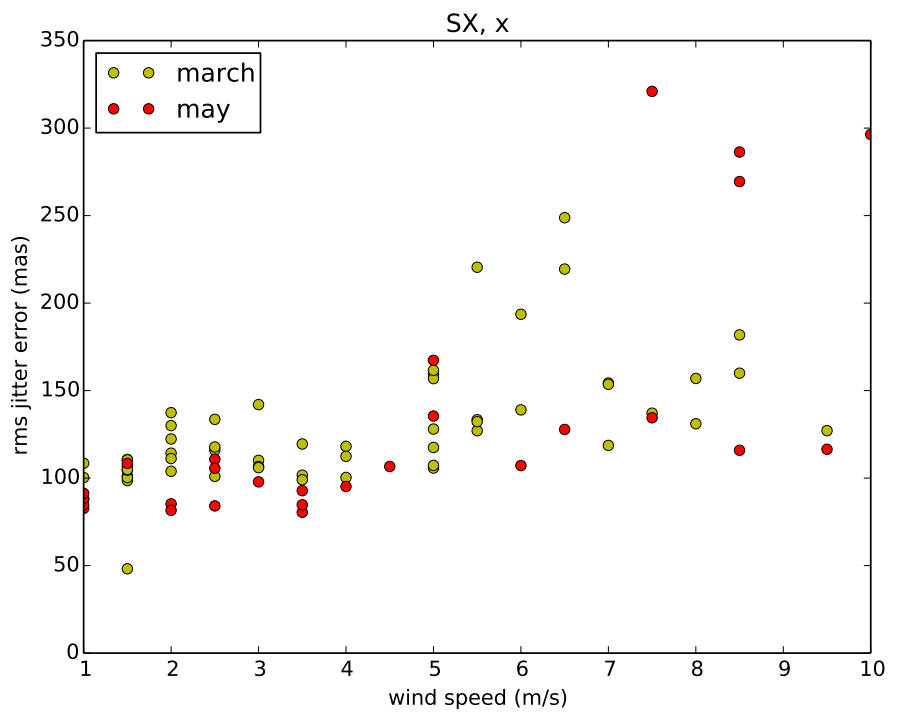

Figure 7: Jitter error before (march) and after (may) the fast link improvement vs. wind speed 


\section{References}

[1] Peter Buschkamp, Walter Seifert, Kai Polsterer, Reiner Hofmann, Hans Gemperlein, Reinhard Lederer, Michael Lehmitz, Vianak Naranjo, Nancy Ageorges, Jaron Kurk, Frank Eisenhauer, Sebastian Rabien, Mathias Honsberg, and Reinhard Genzel. Luci in the sky: performance and lessons learned in the first two years of near-infrared multi-object spectroscopy at the lbt. volume 8446, pages 84465L-84465L-11, 2012.

[2] G. Orban de Xivry, S. Rabien, L. Busoni, W. Gässler, M. Bonaglia, J. Borelli, M. Deysenroth, S. Esposito, H. Gemperlein, M. Kulas, M. Lefebvre, T. Mazzoni, D. Peter, A. Puglisi, W. Raab, G. Rahmer, A. Sivitilli, J. Storm, and J. Ziegleder. First on-sky results with ARGOS at LBT. volume 9909, pages 990936-990936-10, 2016.

[3] Diethard Peter, Wolfgang Gässler, Jose Borelli, and Lothar Barl. Vibration compensation for the ARGOS laser up-link path. 2011.

[4] Diethard Peter, Wolfgang Gässler, Jose Borelli, Lothar Barl, and S. Rabien. Vibration control for the ARGOS laser launch path. volume 8447, pages 84474J-84474J-8, 2012.

[5] Walter Seifert, Immo Appenzeller, Harald Baumeister, Peter Bizenberger, Dominik Bomans, Ralf-Juergen Dettmar, Bernard Grimm, Tom Herbst, Reiner Hofmann, Marcus Juette, Werner Laun, Michael Lehmitz, Roland Lemke, Rainer Lenzen, Holger Mandel, K. Polsterer, RalfRainer Rohloff, A. Schuetze, Andreas Seltmann, Niranjan A. Thatte, Peter Weiser, and Wenli Xu. Lucifer: a multi-mode nir instrument for the lbt. volume 4841, pages 962-973, 2003. 